\title{
Opportunistic scheduling in cellular systems in the presence of non-cooperative mobiles
}

\author{
Veeraruna Kavitha, Eitan Altman, Rachid El-Azouzi and Rajesh Sundaresan \\ Kavitha.Voleti_Veeraruna@sophia.inria.fr, Eitan.Altman@sophia.inria.fr, \\ rachid.elazouzi@univ-avignon.fr, rajeshs@ece.iisc.ernet.in
}

\begin{abstract}
A central scheduling problem in wireless communications is that of allocating resources to one of many mobile stations that have a common radio channel. Much attention has been given to the design of efficient and fair scheduling schemes that are centrally controlled by a base station (BS) whose decisions depend on the channel conditions of each mobile. The BS is the only entity taking decisions in this framework based on truthful information from the mobiles on their radio channel. In this paper, we study the scheduling problem from a game-theoretic perspective in which some of the mobiles may be noncooperative. We model this as a signaling game and study its equilibria. We then propose various approaches to enforce truthful signaling of the radio channel conditions: an approach based on some knowledge of the mobiles' policies, and an approach that replaces this knowledge by a stochastic approximations approach that combines estimation and control. We further identify other equilibria that involve non-truthful signaling.
\end{abstract}

\section{INTRODUCTION}

Short-term fading arises in a mobile wireless radio communication system in the presence of scatterers, resulting in time-varying channel gains. Various cellular networks have downlink shared data channels that use scheduling mechanisms to exploit the fluctuations of the radio conditions (e.g. 3GPP HSDPA [2] and CDMA/HDR [5] or 1xEV-DO [1]). The scheduler design and the obtained gain are predicated on the mobiles sending information concerning the downlink channel gains in a truthful fashion. In a frequencydivision duplex system, the base station (BS) has no direct information on the channel gains, but transmits downlink pilots, and relies on the mobiles' reported values of gains on these pilots for scheduling. A cooperative mobile will truthfully report this information to the BS. A noncooperative mobile will however send a signal that is likely to induce the scheduler to behave in a manner beneficial to the mobile.

Our paper is concerned with game-theoretic analysis of downlink scheduling in the presence of noncooperative mobiles. We consider the BS to be a player as well, and assume that the identity of players that do not cooperate is common knowledge. We model this game as a signaling game which is somewhat uncommon in that there are several players that send signals (the mobiles whose signals correspond to the reported states of the channel) and one follower who reacts to the signal - the BS who decides to whom to allocate the channel. The utility of the BS is assumed to be the social optimum, i.e., the sum of throughputs. The equilibria of this game and the various ways to obtain a truth revealing equilibrium are the focus of our research reported here.

Contribution of the paper: We begin with the case in which BS does not use any extra intelligence to deal with noncooperative mobiles (BS makes scheduling decisions based only the signals from the mobiles). The only Perfect Bayesian Equilibrium (PBE) of the resulting signaling game are the babbling type : the noncooperative mobiles send signals independent of their channel states, and the BS simply ignores them to allocate channels based only on prior channel statistics (Section IV). Fortunately, the BS can use more intelligent strategies to achieve a truth revealing equilibrium hence forth called as TRE. In Section V the BS learns mobiles' signaling statistics, correlates them with the true channel statistics, and punishes the deceivers to obtain a TRE. We next come up with a practical strategy to achieve a TRE in the form of a variant of the proportional fair sharing algorithm (PFA) which elicits truthful signals from mobiles (Section VI). Further, in Section VII, we establish the existence of other equilibria at which the BS improves its utility in comparison to that obtained at a babbling equilibrium; the noncooperative mobiles also improve their utilities over their cooperative shares (utilities at TRE).

Prior work. (1) Proportional fair sharing and other related algorithms were intensely analyzed as applied to the CDMA/HDR system. See [8], [5], [4], [21], [3], [7], [17]. These results are applicable to the 3GPP HSDPA system as well. Kushner \& Whiting [16] analyzed the PFA using stochastic approximation techniques and showed that the asymptotic averaged throughput can be driven to optimize a certain system utility function (sum of logarithms of offsetrates). All the above methods assume that the centralized scheduler has complete information of all relevant quantities.

(2) Mobiles in our setting are more informed than the BS; they have access to their individual channel realization which is private information. If mobiles exploit the ignorance of the BS to their advantage, and the BS is aware of this possibility, we have a signaling game as discussed earlier with several lead players or senders (mobiles) and one follower or receiver (BS). See [15] for an illuminating survey on this topic. Our interest will be in a game with "cheap talk"; this is a signaling game where the sender incurs no cost for his signals [15, Sec. 7]. It is well-known that such games admit a babbling equilibrium. There are situations where other equilibria exist. Interestingly, there are situations where 
cheap talk helps (Farrell [9]), i.e., cheap talk induces a truthrevealing equilibrium while the game with no communication has no equilibrium.

(3) Effect of noncooperative mobiles was recently considered in [14], where the special case of maximum rate algorithm was simulated and shown to improve the noncooperative mobile's throughput by $5 \%$ but decrease the overall system throughput by $20 \%$. Then, observing that the presence of more mobiles in the system resulted in less aggressive signaling, they proposed that the BS advertise the presence of an inflated number of mobiles in the system; the noncooperative mobiles take this advertisement to be true ${ }^{1}$. Nuggehalli et al [18] considered noncooperation by lowpriority latency-tolerant mobiles in an 802.11e LAN setting capable of providing differentiated quality of service. They provide an incentive mechanism in the form of collision free access (CFA) : a guaranteed fraction of allocation to CFA induces truth revelation. Price \& Javidi [20] consider an uplink version of a problem where the mobiles are the informed parties on the valuation of the uplinks (queue state information is available only at the mobile). They provide incentives in the form of allocation on the downlink to induce truth revelation.

Paper outline: After providing a brief system and problem description (Section II and III), we consider signaling game in Section IV. The signaling game admits only babbling equilibria. We then describe in Sections V and VI the two approaches to obtain a truth revealing equilibrium. Other equilibria are identified in Section VII.

\section{PRoblem Formulation}

We consider a wireless network with one base station (BS). There are $M$ mobiles competing for the downlink data channel. Time is divided into small intervals or slots. In each slot one of the $M$ mobiles is allocated the channel. Each mobile $m$ can be in one of the states $h_{m} \in \mathcal{H}_{m}$, where $\mathcal{H}_{m}$ is finite valued. We assume fading characteristics to be independent across the mobiles. Let $\mathbf{h}:=\left[h_{1}, h_{2}, \cdots, h_{M}\right]^{t}$ be the vector of channel gains in a particular slot. The channel gains are distributed according to: $p_{\mathbf{H}}(\mathbf{h})=\prod_{m=1}^{M} p_{H_{m}}\left(h_{m}\right)$, where $\left\{p_{H_{m}} ; m \leq M\right\}$ represents the statistics of the mobile channels. We assume that the mobiles estimate the channel $h_{m}$ perfectly using the pilot signals sent by BS. The mobiles send signals $\left\{s_{m}\right\}$ to BS; these are indications of the channel gains. Some mobiles (with indices $1 \leq m \leq M_{1}$ where $\left.1 \leq M_{1} \leq M\right)$ are assumed to be noncooperative and may signal a better channel condition to grab the channel even when their channel is bad. We assume that signals are chosen from the channel space itself, i.e., $s_{m} \in \mathcal{H}_{m}$ for all mobiles. BS makes a scheduling decision based on signals $\mathbf{s}:=\left[s_{1}, s_{2}, \cdots, s_{M}\right]^{t}$.

Utilities: If the channel is allocated to mobile $m$, it gets a utility $f\left(h_{m}\right)$ which only depends upon its own channel state. An example utility is $f\left(h_{m}\right)=\log \left(1+h_{m} \mathrm{SNR}\right)$ where SNR captures the nominal received signal-to-noise

\footnotetext{
${ }^{1}$ They do not address the possibility of this advertised number being just another signal.
}

ratio under no channel variation. The utility of the BS is taken to be sum of the utilities of all the mobiles. Optimizing the sum utility at the BS results in an efficient solution. It however may be unfair because far-off mobiles may be ignored for they are not likely to contribute to efficiency. In this paper, we mainly study efficient solutions. This is fair only in symmetric mobiles case. Extensions to obtain fairness for asymmetric mobiles are discussed in our next papers.

Common Knowledge : The channel statistics $\left\{p_{H_{m}} ; m \leq M\right\}$ and the information about which mobiles are noncooperative is common knowledge (i.e., known to all the mobiles and the BS). If the BS does not know which mobiles are cooperative, it will treat every mobile as noncooperative. In Sections V to VII wherein the BS estimates either the signal statistics or the average utility of the mobile, the BS can detect the noncooperative mobiles.

\section{System Model}

We now formalize our notation and assumptions : For a set $\mathcal{C}$, let $\mathcal{P}(\mathcal{C})$ be the set of probability measures on $\mathcal{C}$. A policy of mobile $m$ is a function $\left\{\mu_{m}\left(. \mid h_{m}\right)\right\}$ that maps a state $h_{m}$ to an element in $\mathcal{P}\left(\mathcal{H}_{m}\right)$. A policy of the BS is a function $\beta=\{\beta(. \mid \mathbf{s})\}$ that maps each signal $\mathbf{s}$ to an element in $\mathcal{P}(\{1,2, \cdots, M\})$. In later sections this policy can also be a function of other parameters.

The utility of the mobile $m$ depends only upon the true channel $h_{m}$ and the allocation $A$ of the BS, where $A \in$ $\{1, \cdots, M\}$, given by ${ }^{2}: U_{m}\left(s_{m}, h_{m}, A\right)=1_{\{A=m\}} f\left(h_{m}\right)$, while that of BS is $U_{B S}(\mathbf{s}, \mathbf{h}, A)=\sum_{m=1}^{N} U_{m}\left(s_{m}, h_{m}, A\right)$.

Remarks on choice of utility: Even if a mobile signals more than its true value and the BS attempts to transmit at that higher transmitted rate, the actual rate at which the transmission takes place will still be $f\left(h_{m}\right)$. This is reasonable given the following observations. The reported channel is usually subject to estimation errors and delays, an aspect that we do not consider explicitly in this paper. To address this issue, the BS employs a rateless code, i.e., starts at an aggressive modulation and coding rate, gets feedback from the mobile after each transmission, and stops as soon as sufficient number of redundant bits are received to meet the decoding requirements. This incremental redundancy technique supported by hybrid ARQ is already implemented in the aforementioned standards (3GPP HSDPA and 1xEVDO). Then a rate close to the true utility may be achieved.

Define $\quad \mathbf{h}_{-m}:=\left[h_{1}, \cdots, h_{m-1}, h_{m+1}, \cdots, h_{M}\right]$, $p_{H_{-m}}\left(\mathbf{h}_{-m}\right):=\Pi_{j \neq m} p_{H_{j}}\left(h_{j}\right)$ and $\mu_{-m}\left(\mathbf{s}_{-m} \mid \mathbf{h}_{-m}\right):=$ $\Pi_{j \neq m ; j \leq M_{1}} \mu_{j}\left(s_{j} \mid h_{j}\right) \Pi_{j \neq m ; j>M_{1}} \delta\left(h_{j}=s_{j}\right)$ to exclude mobile $m$. Define $\mu(\mathbf{s} \mid \mathbf{h})=\mu_{1}\left(s_{1} \mid h_{1}\right) \mu_{-1}\left(\mathbf{s}_{-1} \mid \mathbf{h}_{-1}\right)$.

Hence the instantaneous utility of mobile $m$, when its channel condition is $h_{m}$, when the mobiles use strategies

\footnotetext{
${ }^{2}$ This is the case if the BS allocates whatever the mobile has signaled for and whenever it has signaled more than or equal to its true value. In the later sections, while developing robust BS policies we will come across the cases, wherein the BS allocates an utility (say $\tilde{u}$ ) which can be different from the requested. In these cases, $U_{m}\left(s_{m}, h_{m}, A\right)=1_{\{A=m\}} \min \left\{\tilde{u}, f\left(h_{m}\right)\right\}$.
} 
$\mu:=\left\{\mu_{m} ; m \leq M_{1}\right\}$, and when the BS uses strategy $\beta$, is

$U_{m}\left(\mu, h_{m}, \beta\right)$

$$
\begin{aligned}
& =\mathbb{E}_{\mathbf{h}_{-m}}\left[\sum_{\mathbf{s}} U_{m}\left(s_{m}, h_{m}, m\right) \beta(m \mid \mathbf{s}) \mu(\mathbf{s} \mid \mathbf{h})\right] \\
& =f\left(h_{m}\right) \mathbb{E}_{\mathbf{h}_{-m}}\left[\sum_{\mathbf{s}} \beta(m \mid \mathbf{s}) \mu(\mathbf{s} \mid \mathbf{h})\right] .
\end{aligned}
$$

Throughout when $\arg \max S$ has more than one element, we write $i=\arg \max S$ to mean $i \in \arg \max S$. By $j:=$ $\arg \max S$ we mean that $j$ is a chosen element of $\arg \max S$.

\section{Signaling Game and Babbling Equilibrium}

The signaling game for downlink scheduling is described as follows: $M_{1}$ mobiles with the lowest indices are the leaders or senders in the signaling game. The BS is the only follower or receiver. The true channel $h_{m}$ represents the true type of leader $m$ with signal $s_{m}$. The policies and utilities of the game are defined in previous paragraphs.

A refinement of NE for such games is a Perfect Bayesian Equilibrium (PBE). This is based on rationale of credible posterior beliefs (Kreps \& Sobel [15, Sec. 5], Sobel [22]).

Definition 4.1: [Posterior beliefs $\pi:=\left\{\pi_{m} ; m \leq M_{1}\right\}$ ] $\pi\left(h_{m} \mid s_{m}\right)$ is the BS's belief of the posterior probability that the mobile's true channel is $h_{m}$ given its signal is $s_{m}$.

Definition 4.2: [Perfect Bayesian Equilibrium (PBE)] A PBE is a strategy profile $\left(\mu_{1}^{*}, \cdots, \mu_{M_{1}}^{*} ; \beta^{*}\right)$ and a posterior belief profile $\pi^{*}$ such that : Given posterior belief profile $\pi_{m}^{*}$, for each signal vector $\mathbf{s}$, the BS chooses $\beta^{*}$ such that

$$
\begin{aligned}
\beta^{*}(\cdot \mid \mathbf{s}) \in & \arg \max _{\gamma \in \mathcal{P}((1, \cdots, M))} \sum_{j>M_{1}} \gamma(j) f\left(s_{j}\right) \\
& +\sum_{j \leq M_{1}} \gamma(j) \sum_{h_{j}} \pi_{j}^{*}\left(h_{j} \mid s_{j}\right) f\left(h_{j}\right) .
\end{aligned}
$$

Given $\beta^{*}$, each mobile $m \leq M_{1}$ chooses $\mu_{m}^{*}$, such that,

$$
\begin{aligned}
& \mu_{m}^{*}\left(\cdot \mid h_{m}\right) \in \arg \max _{\alpha \in \mathcal{P}\left(\mathcal{H}_{m}\right)} \sum_{s_{m} \in \mathcal{H}_{m}} \alpha\left(s_{m}\right)\left[\sum_{\mathbf{s}_{-m}} \beta^{*}(m \mid \mathbf{s})\right. \\
& \left.\sum_{\mathbf{h}_{-m}} p_{H_{-m}}\left(\mathbf{h}_{-m}\right) \mu_{-m}^{*}\left(\mathbf{s}_{-m} \mid \mathbf{h}_{-m}\right) f\left(h_{m}\right)\right] \text { for each } h_{m} \in \mathcal{H}_{m}(2)
\end{aligned}
$$

For each $m \leq M_{1}, s_{m} \in \mathcal{H}_{m}$, the BS updates,

$$
\pi_{m}^{*}\left(h_{m} \mid s_{m}\right)=\frac{p_{H_{m}}\left(h_{m}\right) \mu_{m}^{*}\left(s_{m} \mid h_{m}\right)}{\sum_{h^{\prime} \in \mathcal{H}_{m}} p_{H_{m}}\left(h^{\prime}\right) \mu_{m}^{*}\left(s_{m} \mid h^{\prime}\right)},
$$

if the denominator in (3) is nonzero, and $\pi_{m}^{*}\left(\cdot \mid s_{m}\right)$ is any element in $\mathcal{P}\left(\mathcal{H}_{m}\right)$ otherwise.

In the sequel, we will come across two types of PBE ([22]). The first is the babbling equilibrium where the sender's strategy is independent of its type, and the receiver's strategy is independent of signals. The second is the desirable separating equilibrium where sender sends signals from disjoint subsets of the set of available signals for each type.

We will now show that without any extra intelligence to combat noncooperation, i.e., if the BS schedules based only upon the signals from the mobiles, there exist only babbling equilibria. The following theorem characterizes all the possible PBE (proof given in [11], [12]).

Theorem 1: The above $M_{1}+1$ player signaling game has a PBE of the following type (for all $m$, $\left.h_{m}, s_{m}\right)$ : With $m_{N C}^{*}:=\arg \max _{m \leq M_{1}} \mathbb{E}\left[f\left(h_{m}\right)\right]$, $m_{C}^{*}(\mathbf{s}) \quad:=\arg \max _{m>M_{1}} f\left(s_{m}\right)$ and $m^{*}(\mathbf{s}) \quad:=$ $\arg \max _{m \in m_{N C}^{*} \cup m_{C}^{*}} \mathbb{E}\left[f\left(h_{m}\right) \mid s_{m}\right]$ for all $\mathbf{s}$,

$$
\begin{array}{rcl}
\mu_{m}^{*}\left(s_{m}=s_{m}^{\prime} \mid h_{m}\right) & \text { equals } & \text { any fixed } \mu_{m} \in \mathcal{P}\left(\mathcal{H}_{m}\right), \\
\pi_{m}^{*}\left(h_{m} \mid s_{m}\right) & = & p_{H_{m}}\left(h_{m}\right) \text { and } \\
\beta^{*}(m \mid \mathbf{s}) & \text { equals } & \text { any fixed } \gamma_{\mathbf{s}} \in \mathcal{P}\left(m^{*}(\mathbf{s})\right) .
\end{array}
$$

Further, these are the only type of PBE for this game.

In the above equilibrium, the (noncooperative) mobile's strategy is to send a signal independent of the channel value, and the BS's strategy is to ignore the signals from the noncooperative mobiles. Hence it is a babbling equilibrium. Further, possibility of a 'separating' PBE (TRE) is ruled out.

The scheduling policy of the babbling PBE of Theorem 1 results in the following utility for the BS :

$$
\begin{array}{r}
U_{c o p}^{*}:=\mathbb{E}_{\mathbf{h}_{>M_{1}}}\left[\max \left\{\max _{m>M_{1}} f\left(h_{m}\right), \max _{m \leq M_{1}} \mathbb{E}\left[f\left(h_{m}\right)\right]\right\}\right] \\
\text { where } \mathbf{h}_{>M_{1}}:=\left[\begin{array}{llll}
h_{M_{1}+1}, & h_{M_{1}+2}, & \cdots, & h_{M}
\end{array}\right]^{T} .
\end{array}
$$

It is the best utility that the BS can get using only information from cooperative mobiles and the statistics of noncooperative mobiles. Note that the BS uses very little information here to attain $U_{c o p}^{*}$ (only the signals and prior information). To do better, BS has to use extra intelligence. In coming sections we obtain the desirable TRE via two different approaches.

\section{A TRE: By 'Predicting' Signal Statistics}

In this section, we assume that the BS is able to 'predict' the statistics of the signals generated by all the mobiles, perhaps based on observations of past behavior, before the game is executed. With $p_{S_{m}}(s):=\sum_{h} \mu_{m}(s \mid h) p_{H_{m}}(h)$ for all $s \in \mathcal{H}_{m}$ and for all $m$, let $p_{\mathbf{S}}:=\left(p_{S_{1}}, p_{S_{2}}, \cdots, p_{S_{M}}\right)$ represent the tuple of the signal probabilities of all the mobiles. The BS policy is now a probability measure over the set of mobiles for each signal vector $\mathbf{s}$ and each $p_{\mathbf{S}}$ and hence is given by $\beta\left(. \mid \mathbf{s}, p_{\mathbf{s}}\right)$. This results in a completely different game and we obtain a TRE using this new policies.

The new game is still an incomplete information game but is no more a signaling game. We now consider a simple $M_{1}+1$ player strategic form game. The rest of the details of the game, i.e., all the utilities and the policies of the mobiles, remain the same as that in the previous sections.

Consider the following 'predictive' policy $\beta_{p}^{*}$ of the BS: $B S$ allocates the channel to the mobile with maximum signaled rate if its signal statistics are same as its true channel statistics. If not the BS allocates the channel to the cooperative mobile with maximum signaled rate among the cooperative mobiles.

Policy $\beta_{p}^{*}$ at BS and the truth revealing signals (i.e., $\mu_{m}^{*}(s \mid h)=\delta(s=h)$ at all the mobiles forms a NE, i.e., a TRE, because: If a mobile generates a noncooperative 
signal such that the signal statistics remain the same as the true channel statistics, he will have to lose the channel in one of his good states for a gain of the channel in one of his bad states. At a TRE, the BS obtains the following utility :

$$
U_{\text {max }}^{*}:=\mathbb{E}_{\mathbf{h}}\left[\max _{m \leq M} f\left(h_{m}\right)\right] \text {. }
$$

It is the utility that the BS can get by using the truthful information from all the mobiles. It is easy to see that this is the maximum utility that the BS can get and hence is also the maximum utility of the BS at equilibrium among all the possible NE.

\section{STOCHASTIC APPROXIMATION}

In the previous section a TRE is obtained by assuming the availability of perfect 'signal statistics' estimates at the BS. Any estimation procedure will have errors and it is of interest to study the impact of these errors on the desired NE. In this section we directly estimate the average (signaled) throughput of all the mobiles and use these estimates to obtain more realistic (truth revealing) policies at the BS.

A policy based on stochastic approximation is proposed. It operates over several time slots and estimates parameters online. Such an approach is also well-suited to track changes in model parameters. Our policy has a "corrective" feature because the BS continually (i) estimates the average throughput that each mobile gets; (ii) estimates the excess utility that each mobile accumulates beyond its cooperative share (its share in a cooperative setting); (iii) applies a "corrective" term based on the excess utility. The resulting estimates are then used to make scheduling decisions.

We define an appropriate $M_{1}+1$-player strategic form game and show that a "corrective" policy at the BS, along with the truth-revealing strategies at mobiles, forms an $\epsilon$ Nash equilibrium, and the policy is thus near-TRE ${ }^{3}$.

We begin by first defining the policies and utilities of the players involved in the game (some notations of this section are different from the rest of the paper). The policy of a BS is a time-varying function whose action at time $k$ depends on the signals sent by the mobiles up to and including time $k$. Throughout this section, we model channel gains as bounded random variables taking values on a continuum and satisfying assumption A.3 given below. The policy of a mobile $m$ for $m \leq M_{1}$ is given by a measurable signal map $s_{m}: \mathcal{H}_{m} \mapsto$ $\mathcal{H}_{m}$ that satisfies assumption A.2 given below. For the sake of uniformity we define $s_{m}\left(h_{m}\right)=h_{m}$ for $m>M_{1}$, i.e., the cooperative mobiles report the true channel. Thus, the signaled utility at time $k$ is $f\left(s_{m}\left(h_{m, k}\right)\right) ; m \leq M$, while the true utility is $f\left(h_{m, k}\right) ; m \leq M$.

Next we define the utilities of mobiles, BS. Let $\phi_{m, k}$ be the slot-level utility derived by mobile $m$ in slot $k$. Note that $0 \leq \phi_{m, k} \leq f\left(h_{m, k}\right)$ and $\phi_{m, k}=0$ if the channel is not assigned to mobile $m$ in slot $k$. We then set for all $m \leq M$,

$$
U_{m}=\lim _{k \rightarrow \infty} \frac{1}{k} \sum_{l \leq k} \phi_{m, l} \quad \text { and } U_{B S}=\sum_{m=1}^{M} U_{m}
$$

${ }^{3}$ This $\epsilon$-Nash equilibrium is in fact slightly stronger equilibrium than NE. if all the limits exist. In a TRE, the BS achieves the maximum sum utility $U_{\text {max }}^{*}$ given in (5) while the $m$ th mobile gets

$$
U_{m}^{*}=\theta_{m}^{0}:=\mathbb{E}_{\mathbf{h}}\left[f\left(h_{m}\right) 1_{\left\{f\left(h_{m}\right) \geq f\left(h_{j}\right) \text { for all } j \neq m\right\}}\right] .
$$

Note that BS can calculate $\Theta^{0}:=\left(\theta_{1}^{0}, \cdots, \theta_{M}^{0}\right)$ with its available knowledge. We now propose the following iterative "corrective" scheduling algorithm at the BS:

$$
\begin{aligned}
\theta_{m, k+1} & =\theta_{m, k}+\epsilon_{k}\left(\tilde{f}_{m, k+1} I_{m, k+1}-\theta_{m, k}\right) \\
\tilde{f}_{m, k+1} & =f\left(s_{m}\left(h_{m, k+1}\right)\right)-\left(\theta_{m, k}-\theta_{m}^{0}\right) \Delta \\
I_{m, k+1} & \left.=1_{\left\{\tilde{f}_{m, k+1} \geq \tilde{f}_{j, k+1}\right.} \text { for all } j \neq m\right\}^{1}\left\{\tilde{f}_{m, k+1} \geq 0\right\}
\end{aligned}
$$

with initial conditions $\theta_{m, 0}=\theta_{m}^{0}$ for all $m$. The BS for each mobile i) tracks average reported utility via $\theta_{m, k}$, ii) computes excess utility $\theta_{m, k}-\theta_{m}^{0}$, relative to the mobiles cooperative share in a TRE, iii) subtracts the excess from the instantaneous signaled utility after magnification by $\Delta$, and uses the updated values to make a current scheduling decision. The choice of $\Delta$ depends on $\epsilon$.

If BS schedules mobile $m$ in slot $k$, the latter gets a utility

$$
\bar{f}_{m, k}:=\min \left\{\tilde{f}_{m, k}, f\left(h_{m, k}\right)\right\} .
$$

Indeed, if $\tilde{f}_{m, k}<0$ for the selected mobile, no transmission is made. If $\tilde{f}_{m, k}<f\left(h_{m, k}\right)$, transmission is made at lesser rate to get a slot-level utility of $\tilde{f}_{m, k}$. If $\tilde{f}_{m, k} \geq$ $f\left(h_{m, k}\right)$, our remarks on utilities in Section III provide a slot-level utility of $f\left(h_{m, k}\right)$. Thus the achieved utility in slot $k$ is (8). Consequently, utility for mobile $m$ is $U_{m}=$ $\lim _{k \rightarrow \infty} \frac{1}{k} \sum_{l=1}^{k} \bar{f}_{m, l} I_{m, l}$, which can be rewritten as limit of,

$$
\bar{\theta}_{m, k+1}=\bar{\theta}_{m, k}+\epsilon_{k}\left(\bar{f}_{m, k+1} I_{m, k+1}-\bar{\theta}_{m, k}\right),
$$

Thus the mobile and the BS utilities are

$$
U_{m}=\lim _{k \rightarrow \infty} \bar{\theta}_{m, k} \text { for all } m \leq M \text { and } U_{b s}=\sum_{m} U_{m}
$$

Analysis of the policy: Let $S=\left(s_{1}, s_{2}, \cdots s_{M}\right)$ represent a strategy profile. (last $M-M_{1}$ maps are identity maps).

Assumptions : We assume the following :

A.1 The function $f$ is bounded and invertible. Both the functions $f, f^{-1}$ are continuously differentiable;

A.2 The signal maps $s_{m}$ are such that the density of the random variables $s_{m}\left(h_{m}\right)$ are bounded;

A.3 The processes $\left\{h_{m, k}\right\}_{k \geq 1}$ are stationary Markov chains for each mobile $m$, and independent across mobiles. The random variable $h_{m, 1}$ is a bounded random variable with bounded density for each $m$.

We use ODE approximation theory ([16], [6] etc.) to analyze the utilities (10) and obtain optimality properties of (6). Define $\Theta_{k}:=\left(\theta_{1, k}, \cdots, \theta_{m, k}\right), \bar{\Theta}_{k}:=\left(\bar{\theta}_{1, k}, \cdots, \bar{\theta}_{m, k}\right)$ and $\Psi_{k}:=\left(\Theta_{k}, \bar{\Theta}_{k}\right)$. Define $t(r):=\sum_{k=0}^{r} \epsilon_{k}, m(n, T):=$ $\arg \max _{r \geq n}\{t(r)-t(n) \leq T\}$. Let $\Psi\left(t, t_{0},\left(\Theta_{0}, \bar{\Theta}_{0}\right)\right)$ represent the solution of the pair of ODEs,

$$
\begin{aligned}
& \dot{\Theta}(t)=H^{S}(\Theta(t))-\Theta(t) ; \quad \Theta\left(t_{0}\right)=\Theta_{0} \\
& \dot{\bar{\Theta}}(t)=\bar{H}^{S}(\Theta(t))-\bar{\Theta}(t) ; \quad \bar{\Theta}\left(t_{0}\right)=\bar{\Theta}_{0}
\end{aligned}
$$




$$
\begin{aligned}
& H_{m}^{S}(\Theta):=\mathbb{E}_{\mathbf{h}}\left[\tilde{f}_{m}^{S}\left(h_{m}, \theta_{m}\right) I_{m}^{S}(\mathbf{h}, \Theta)\right] \\
& \bar{H}_{m}^{S}(\Theta):=\mathbb{E}_{\mathbf{h}}\left[\bar{f}_{m}^{S}\left(h_{m}, \theta_{m}\right) I_{m}^{S}(\mathbf{h}, \Theta)\right] \\
& \tilde{f}_{m}^{S}\left(h_{m}, \theta_{m}\right):=f\left(s_{m}\left(h_{m}\right)\right)-\left(\theta_{m}-\theta_{m}^{0}\right) \Delta \\
& I_{m}^{S}(\mathbf{h}, \Theta):=1_{\left\{\tilde{f}_{m}^{S}\left(h_{m}, \theta_{m}\right) \geq \tilde{f}_{j}^{S}\left(h_{j}, \theta_{j}\right) \text { for all } j \neq m\right\}} \\
& 1_{\left\{\tilde{f}_{m}^{S}\left(h_{m}, \theta_{m}\right)>0\right\}} \\
& \bar{f}_{m}^{S}\left(h_{m}, \theta_{m}\right):=\min \left\{\tilde{f}_{m}^{S}\left(h_{m}, \theta_{m}\right), f\left(h_{m}\right)\right\} .
\end{aligned}
$$

We have the approximation theorem (proof in [11], [12]).

Theorem 2: The ODEs have unique solution for any finite time $T$. Assume A.1-A. 3 and $\left\{\epsilon_{k}=(k+1)^{-1}\right\}$. Fix any $T>0, \delta>0, \mathbf{h}$, and let $\left(\mathbf{h}_{n}, \Psi_{n}\right)$ be initialized to $(\mathbf{h}, \Psi)=(\mathbf{h},(\Theta, \bar{\Theta}))$. Let $P_{n: \mathbf{h}, \Psi}$ denote the distribution of $\left\{\left(\mathbf{h}_{n+k}, \Psi_{n+k}\right)\right\}_{k \geq 0}$ with initializations $\mathbf{h}_{n}=h, \Psi_{n}=\Psi$. Then as $n \rightarrow \infty$

$P_{n: \mathbf{h}, \Psi}\left\{\sup _{\{n \leq r \leq m(n, T)\}}\left|\Psi_{r}-\Psi(t(r), t(n), \Psi)\right| \geq \delta\right\} \rightarrow 0$

uniformly for all $\Psi \in Q_{1}$, where $Q_{1}$ is any compact set. $\square$

By the above theorem, trajectory $\left\{\bar{\theta}_{m, k} ; m \leq M\right\}$ is approximated by the solution of ODE (12). Hence we analyze the utilities (10) using the limits (attractors) of the ODE.

For any strategy profile $S$, any attractor $\Theta^{*}$ of the ODE (11) satisfies

$$
\begin{aligned}
\theta_{m}^{*}-\theta_{m}^{0} & =\frac{\mathbb{E}_{\mathbf{h}}\left[f\left(s_{m}\left(h_{m}\right)\right) I_{m}^{S}\left(\mathbf{h}, \Theta^{*}\right)\right]-\theta_{m}^{0}}{1+\Delta E\left[I_{m}^{S}\left(\mathbf{h}, \Theta^{*}\right)\right]} \\
& \leq \frac{\left(\max _{h} f(h)\right) \mathbb{E}_{\mathbf{h}}\left[I_{m}^{S}\left(\mathbf{h}, \Theta^{*}\right)\right]}{1+\Delta E\left[I_{m}^{S}\left(\mathbf{h}, \Theta^{*}\right)\right]} \leq o(1 / \Delta) .
\end{aligned}
$$

Any attractor of (12) satisfies $\bar{\theta}_{m}^{*}=\bar{H}_{m}^{S}\left(\Theta^{*}\right) \leq \theta_{m}^{*}$. Thus,

$$
U_{m} \approx \bar{\theta}_{m}^{*} \leq \theta_{m}^{0}+o(1 / \Delta) .
$$

Let $S=I$, the truth revealing profile $\left(s_{m}\left(h_{m}\right)=h_{m}\right.$ for all $h_{m}, m$ ). Clearly $\Theta^{0}$ is a zero of RHS of ODEs (11), (12). One can easily show that it will indeed be an attractor by showing that the derivative of $H^{S}(\Theta)-\Theta$ is negative definite near $\Theta^{0}$. Hence $U_{m}=\theta_{m}^{0}$ when $S=I$ for all $m$.

From (13) none of the mobiles, no matter what strategy they use or no matter what strategy the other mobiles use, can gain more than $\theta_{m}^{0}$. This along with para above implies that the 'corrective' policy (with appropriately large $\Delta$ ) of $B S$ together with the truth-revealing signals at all the mobiles forms an $\epsilon$-Nash equilibrium.

Some further Remarks : Note for large values of $\Delta$,

$$
\begin{aligned}
\left(\theta_{m}^{*}-\theta_{m}^{0}\right) \Delta & =\frac{\Delta\left(\mathbb{E}_{\mathbf{h}}\left[f\left(s_{m}\left(h_{m}\right)\right) I_{m}^{S}\left(\mathbf{h}, \Theta^{*}\right)\right]-\theta_{m}^{0}\right)}{1+\Delta E\left[I_{m}^{S}\left(\mathbf{h}, \Theta^{*}\right)\right]} \\
& \approx \frac{\mathbb{E}_{\mathbf{h}}\left[f\left(s_{m}\left(h_{m}\right)\right) I_{m}^{S}\left(\mathbf{h}, \Theta^{*}\right)\right]-\theta_{m}^{0}}{E\left[I_{m}^{S}\left(h, \theta^{*}\right)\right]}
\end{aligned}
$$

which can be significant but is bounded (independently of $\Delta$ ) because of the boundedness of $f$. Now, if any mobile reports much more than its true value, i.e., if $f\left(h_{m}\right) \ll f\left(s_{m}\left(h_{m}\right)\right)$ for significant values of $h_{m}$, and if in fact it is large enough such that $f\left(h_{m}\right) \ll f\left(s_{m}\left(h_{m}\right)\right)-\left(\theta_{m}^{*}-\theta_{m}^{0}\right) \Delta$ then,

$$
U_{m} \ll \mathbb{E}_{\mathbf{h}}\left[\left(f\left(s_{m}\left(h_{m}\right)\right)-\left(\theta_{m}^{*}-\theta_{m}^{0}\right) \Delta\right) I_{m}^{S}\left(\mathbf{h}, \Theta^{*}\right)\right]=\theta_{m}^{*} \text {. }
$$

Hence $U_{m} \ll \theta_{m}^{0}$, i.e., that particular mobile's output is much lesser than $\theta_{m}^{0}$, its own cooperative share. Hence the mobile which deviates the most from true values gets the least share.

Examples : In Figure 1 we consider an example with two identical and cooperative mobiles, which reinforces that the ODE attractors will be good approximations for the time limits of almost all the trajectories of the true utility adaptation (9). Let $f_{Z}\left(z ; \sigma^{2}\right)=z e^{-z^{2} / 2 \sigma^{2}}, z \geq 0$ represent the density of the Rayleigh distributed random variable $Z\left(\sigma^{2}\right)$. The channel gains of both the mobiles are conditional Rayleigh distributed, i.e., for both $m=1,2$ $h_{m} \sim f_{Z}(z ; 3) 1_{\{z \leq 2\}} d z / \operatorname{Prob}(Z(3) \leq 2)$. The utilities used throughout this sub section are the achievable rates $f(h)=$ $\log (1+h)$. We plot two independent trajectories/ sample paths of both the mobiles, $\left\{\bar{\theta}_{m, k}\right\}_{m=1,2} \quad k \geq 1$ initialized away from their cooperative shares $\theta_{1}^{0}=\theta_{2}^{0}=0.456$. We set $\Delta=$ 9000. All the trajectories converge close to the attractors of the ODE thus corroborating theory.

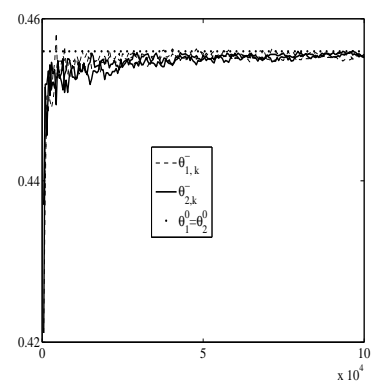

Fig. 1. Accuracy : ODE attractors approximating time limits of (9).

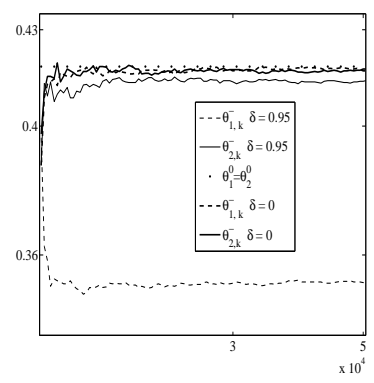

Fig. 2. Robust SA based BS Policy in the presence of noncooperation
In Figure 2 we illustrate the robustness of our BS policy. We consider two symmetric mobiles now with $h_{m} \sim$ $f_{Z}(z ; 1) 1_{\{z \leq 2\}} d z / \operatorname{Prob}(Z(1) \leq 2)$. The first mobile, can be noncooperative with $s_{1}(h)=h+(2-h) \delta$. We plot two sets of trajectories, one (thicker curves) for all cooperative behavior $(\delta=0)$ while the corresponding set of thinner curves belong to the case where the first mobile is noncooperative with $\delta=0.95$ with $\Delta=9000$. All the cooperative curves converge towards the cooperative share (same one for both the mobiles as it is a symmetric situation). While, the true utility of the noncooperative mobile (mobile 1), converges to a value less than the cooperative share, confirming the theory of the previous sections.

The trajectories corresponding to the reported rate $\left\{\theta_{m, k}\right\}$ (6) are not plotted as they converge in all cases close to the cooperative shares $\theta_{m}^{0}$ and they do it much more faster than the true rate trajectories. Hence, they do not convey much information, but just mess up the plots.

\section{EXISTENCE OF OTHER NASH EQUiLIBRIA}

We obtained two types of NE till now. Under the first equilibrium (babbling equilibrium of Theorem 1) BS schedules only using the signals from the cooperative mobiles and the channel statistics of the noncooperative mobiles. The BS utility is the minimum among all the possible equilibrium utilities and equals $U_{c o p}^{*}$ given in (4).

The other type of NE (equilibria of Sections V, VI), are the truth revealing equilibria (TRE). BS achieved these equilibria 
by using ITR (incentives for truth revealing) policies. When in a TRE, the BS schedules using the true channel information of all the mobiles. BS now achieves the maximum possible equilibrium utility $U_{\max }^{*}$ given in (5).

Clearly $U_{c o p}^{*} \leq U_{\text {max }}^{*}$. This raises a natural question about the existence of other NE with BS's equilibrium utility taking any value in the interval $\left[U_{c o p}^{*}, U_{\max }^{*}\right]$. In this section we continue with the 'predictive' policies, $\beta\left(. \mid \mathbf{s}, p_{\mathbf{S}}\right)$, of Section $\mathrm{V}$ and investigate the existence of other NE in Theorem 3.

Let $E^{\mu_{m}}\left[f\left(h_{m}\right) \mid s_{m}\right]$ represent the conditional expectation of the mobile's utility conditioned on the signal $s_{m}$ when mobile $m$ uses strategy $\mu^{m}$, i.e., for every $s_{m} \in \mathcal{H}_{m}$ define

$$
\begin{aligned}
E^{\mu_{m}} & {\left[f\left(h_{m}\right) \mid s_{m}\right] } \\
& :=\sum_{h_{m} \in \mathcal{H}_{m}} \frac{p_{H_{m}}\left(h_{m}\right) \mu_{m}\left(s_{m} \mid h_{m}\right)}{\sum_{\tilde{h}_{m} \in \mathcal{H}_{m}} p_{H_{m}}\left(\tilde{h}_{m}\right) \mu_{m}\left(s_{m} \mid \tilde{h}_{m}\right)} f\left(h_{m}\right) .
\end{aligned}
$$

With this, the payoff for mobile $m$ is,

$$
U_{m}\left(\mu_{m}, \beta\right)=\mathbb{E}_{\mathbf{s}}\left[\beta\left(m \mid \mathbf{s}, p_{\mathbf{S}}\right) E^{\mu_{m}}\left[f\left(h_{m}\right) \mid s_{m}\right]\right] .
$$

In the above, $\mathbb{E}_{\mathbf{s}}$ represents the expectation w.r.t. $p_{\mathbf{S}}$.

Given $p_{\mathbf{S}}$, let $\mu^{*}$ (or more appropriately $\mu^{*}\left(p_{\mathbf{S}}\right)$ ) represent 'best' mobile strategy that gives for every $h^{i}, h^{j} \in$ $\mathcal{H}_{m}$ and for all $m \leq M$

$$
\begin{aligned}
f\left(h^{i}\right) & \geq f\left(h^{j}\right) \\
& \Longrightarrow E^{\mu_{m}^{*}}\left[f\left(h_{m}\right) \mid s_{m}=h^{i}\right] \geq E^{\mu_{m}^{*}}\left[f\left(h_{m}\right) \mid s_{m}=h^{j}\right] .
\end{aligned}
$$

Construction of $\mu^{*}$ : Consider mobile 1 without loss of generality. Let $\mathcal{H}_{1}=\left\{h^{1}, h^{2}, \cdots, h^{N_{1}}\right\}$ and assume $f\left(h^{1}\right) \geq f\left(h^{2}\right) \geq \cdots \geq f\left(h^{N_{1}}\right)$. In the following few lines we leave subscript 1 to improve readability i.e., the random variables $h_{1}, s_{1}$ etc. are represented by $h, s$ etc. Strategy $\mu_{1}^{*}$ is defined in a iterative way.

We will first define $\left\{\mu_{1}^{*}\left(s=h^{1} \mid h\right) ; h \in \mathcal{H}_{1}\right\}$, i.e., the conditional probabilities of declaring to be in its best state $h^{1}$ by mobile 1 when it is actually in any arbitrary state $h \in \mathcal{H}_{1}$. Find the minimum index $j_{1}^{*}$ such that probability of the channel to be in one of the top $j_{1}^{*}$ states is greater than or equal to $p_{S_{1}}\left(h^{1}\right)$, i.e., let, $j_{1}^{*}:=\arg \min _{j}\left\{\sum_{i=1}^{j} p_{H_{1}}\left(h^{i}\right) \geq p_{S_{1}}\left(h^{1}\right)\right\}$. Declare state $h^{1}$ whenever the true channel is one among the top $j_{1}^{*}-1$ states, i.e., for all $h$ with $f(h)>f\left(h^{j_{1}^{*}}\right)$, set $\mu_{1}^{*}(s=$ $\left.h^{1} \mid h\right):=1$. When $h=h^{j_{1}^{*}}$ signal to be in best state $h^{1}$, for a fraction of time, where the fraction is chosen such that the overall probability of signal $s=h^{1}$ will be equal to $p_{S_{1}}\left(h^{1}\right)$, i.e., $\mu_{1}^{*}\left(s=h^{1} \mid h=h^{j_{1}^{*}}\right)=\frac{p_{S_{1}}\left(h^{1}\right)-\sum_{l<j_{1}^{*}} p_{H_{1}}\left(h^{l}\right)}{p_{H_{1}}\left(h^{j_{1}^{*}}\right)}$. Set $\mu_{1}^{*}\left(s=h^{1} \mid h^{i}\right)=0$ whenever $i>j_{1}^{*}$.

Now we define $\left\{\mu_{1}^{*}\left(s=h^{2} \mid h\right) ; h \in \mathcal{H}_{1}\right\}$, i.e., the conditional probabilities of declaring to be in the second best state $h^{2}$ by mobile 1 , when in any arbitrary state $h \in \mathcal{H}_{1}$. Let $j_{2}^{*}:=\arg \min _{j}\left\{\sum_{l=1}^{j} p_{H_{1}}\left(h^{l}\right)-p_{S_{1}}\left(h^{1}\right) \geq p_{S_{1}}\left(h^{2}\right)\right\}$. Now define (the below definitions are for $j_{2}^{*}>j_{1}^{*}$. If not one can appropriately modify the definitions.),

$$
\begin{aligned}
\mu_{1}^{*}\left(s=h^{2} \mid h^{j_{1}^{*}}\right) & =1-\mu_{1}^{*}\left(s=h^{1} \mid h^{j_{1}^{*}}\right), \\
\mu_{1}^{*}\left(s=h^{2} \mid h^{i}\right) & =1 \text { whenever } j_{1}^{*}<i<j_{2}^{*}, \\
\mu_{1}^{*}\left(s=h^{2} \mid h^{j_{2}^{*}}\right) & = \\
\frac{p_{S_{1}}\left(h^{1}\right)+p_{S_{1}}\left(h^{2}\right)-\sum_{l<j_{2}^{*}} p_{H_{1}}\left(h^{l}\right)}{p_{H_{1}}\left(h_{2}^{j_{2}^{*}}\right)} & 0 \text { for the remaining } h .
\end{aligned}
$$

Note that with the above definitions

$$
E^{\mu_{1}^{*}}\left[f\left(h_{1}\right) \mid s=h^{1}\right] \geq f\left(h^{j_{1}^{*}}\right) \geq E^{\mu_{1}^{*}}\left[f\left(h_{1}\right) \mid s=h^{2}\right] .
$$

Continue in the same way to obtain

$$
\begin{array}{r}
E^{\mu_{1}^{*}}\left[f\left(h_{1}\right) \mid s=h^{1}\right] \geq E^{\mu_{1}^{*}}\left[f\left(h_{1}\right) \mid s=h^{2}\right] \geq \\
\cdots \geq E^{\mu_{1}^{*}}\left[f\left(h_{1}\right) \mid s=h^{N_{1}}\right] .
\end{array}
$$

By Result 1 given below, if mobile 1 uses any other strategy $\mu_{1}$ resulting again in the same signal probability $p_{S_{1}}$ of $p_{\mathbf{S}}$ while all other mobiles use their 'best' strategy and the BS uses policy $\beta\left(m \mid \mathbf{s}, p_{\mathbf{S}}\right):=1_{\left\{m=\arg \max _{m} \mathbb{E}\left[f\left(h_{m}\right) \mid s_{m}\right]\right\}}$, then $U_{1}\left(\mu_{1}^{*}, \beta\right) \geq U_{1}\left(\mu_{1}, \beta\right)$. These strategies are called 'best' because with these, the mobile $m$ gets the best payoff for masquerading a signal probability $p_{S_{m}}$.

For $m>M_{1}$, we set $\mu^{*}\left(h_{m} \mid s_{m}\right)=1_{\left\{h_{m}=s_{m}\right\}}$. With the help of 'best' strategies we obtain the existence of other NE:

Theorem 3: For every signal probability tuple $\bar{p}_{\mathbf{S}}$ with the 'best' strategies $\bar{\mu}^{*}$ such that,

$$
\begin{aligned}
U_{c o p}^{*} & \leq \mathbb{E}_{\mathbf{h}, \mathbf{s}}\left[f\left(h_{m^{*}}\right)\right] \text { with } \\
m^{*} & :=\arg \max _{1 \leq m \leq M} E^{\bar{\mu}_{m}^{*}}\left[f\left(h_{m}\right) \mid s_{m}\right],
\end{aligned}
$$

the ordered pair $\left(\bar{\mu}^{*}, \bar{\beta}^{*}\right)$ is a Nash equilibrium where the feedback policy $\bar{\beta}^{*}$ of the BS is given by following:

Let $\mu=\left(\mu_{1}, \mu_{2}, \cdots \mu_{M_{1}}\right)$ be any arbitrary signaling policy of the mobiles and let $p_{\mathbf{S}}=\left\{p_{S_{m}} ; m \leq M_{1}\right\}$ be the signaling probabilities resulting from these actions of the noncooperative mobiles. Define,

$$
q_{m}\left(p_{\mathbf{S}}, s_{m}\right):=\min \left\{1, \frac{\bar{p}_{S_{m}}\left(s_{m}\right)}{p_{S_{m}}\left(s_{m}\right)}\right\} \text { for all } s_{m} \in \mathcal{H}_{m},
$$

and for all $m \leq M_{1}$. For $m>M_{1}$ define $q_{m}(\cdot, \cdot)=$ 1. For any given signal vector $\mathbf{s}$ define, $m_{1}^{*}(\mathbf{s}):=$ $\arg \max _{m \leq M} \quad E^{\bar{\mu}_{m}^{*}}\left[f\left(h_{m}\right) \mid s_{m}\right]$ (the best among all the mobiles) and $m_{2}^{*}(\mathbf{s}):=\arg \max _{m>M_{1}} f\left(s_{m}\right)$ (best among cooperative mobiles). Then define,

$$
\begin{aligned}
\bar{\beta}^{*}\left(m \mid \mathbf{s}, p_{\mathbf{S}}\right) & =0 \text { for all } m \neq m_{1}^{*}, m_{2}^{*} \\
\bar{\beta}^{*}\left(m_{1}^{*} \mid \mathbf{s}, p_{\mathbf{S}}\right) & =q_{m_{1}^{*}}\left(p_{\mathbf{S}}, s_{m_{1}^{*}}\right) \\
\bar{\beta}^{*}\left(m_{2}^{*} \mid \mathbf{s}, p_{\mathbf{S}}\right) & =\left(1-q_{m_{1}^{*}}\left(p_{\mathbf{S}}, s_{m_{1}^{*}}\right)\right) .
\end{aligned}
$$

Proof : If all the noncooperative mobiles are fixed with signaling policy $\bar{\mu}^{*}$ then the signaling probabilities will be given by $\bar{p}_{\mathbf{S}}$ and we have, $q_{m}\left(\bar{p}_{\mathbf{S}}, s_{m}\right)=1$ for all $s_{m} \in \mathcal{H}_{m}$ and for all $m \leq M$. Hence $\bar{\beta}^{*}\left(m \mid \mathbf{s}, \bar{p}_{\mathbf{S}}\right)=1_{\left\{m=m_{1}^{*}(\mathbf{s})\right\}}$.

From (14), the total payoff of the BS with signal probabilities fixed at $\bar{p}_{\mathbf{S}}$, when it uses some arbitrary channel 
allocation say $\beta(. \mid \mathbf{s})$, is given by,

$$
U_{B S}=\mathbb{E}_{\mathbf{s}}\left[\sum_{m=1}^{M} \beta(m \mid \mathbf{s}) \mathbb{E}^{\bar{\mu}_{m}^{*}}\left[f\left(h_{m}\right) \mid s_{m}\right]\right] .
$$

Clearly, the BS achieves the maximum with $\bar{\beta}^{*}$.

Say BS uses the policy $\bar{\beta}^{*}$. Without loss of generality assume mobile 1 unilaterally deviates from strategy $\bar{\mu}_{1}^{*}$ and signals instead using $\mu_{1}$ such that the signal probabilities remain the same. Then by the Result 1 mobile 1 gets lesser than before. If now $\mu_{1}$ is such that even the signal probabilities are different from $\bar{p}_{S_{1}}$ then the payoff of the mobile 1 is further reduced as is seen from (16), as now it is possible that $q_{1}\left(\mu_{1}, s_{1}\right)<1$ for some values (note that $\left(1-q_{1}\left(\mu_{1}, s_{1}\right)\right)$ fraction of the time channel is allocated to a cooperative mobile) and the rest steps are as in the proof of Result 1 stated next.

We would like to emphasize here that the BS calculates $m_{1}^{*}, m_{2}^{*}$ of the above theorem using $\bar{\mu}^{*}$ irrespective of the strategies actually used at the mobiles.

Result 1: Say all mobiles other than 1 use their 'best' strategies, i.e., mobiles $m$ with $m>1$ use strategy $\bar{\mu}_{m}^{*}$. Also assume that BS uses the policy in(16). The payoff of mobile 1 is maximized with its 'best' strategy $\bar{\mu}_{1}^{*}$, when its signal probabilities are restricted to $\bar{p}_{S_{1}}$.

The proof of this Result uses similar constructions as before and is available in [11], [12].

\section{CONCLUDING REMARKS}

We studied centralized downlink transmissions in a cellular network in the presence of noncooperative mobiles. We modeled this as a signaling game with several players controlling signals and where the BS serves as follower. In absence of extra intelligence, only babbling equilibrium is obtained, at which both the BS and the noncooperative players make no use of the signaling opportunities. We then proposed two approaches to obtain an efficient equilibrium (TRE), both of which required extra intelligence from the BS but resulted in the mobiles signaling truthfully. We further showed the existence of other non efficient equilibria at which a noncooperative mobile achieves a better utility while the BS achieves better utility than that at a babbling equilibrium but a lower one than that at a TRE.

We see several avenues open for further research on scheduling under noncooperation. We recall that we assumed that a player is either cooperative or not. What if the player can choose? Preliminary research show that there is no clear answer: it depends on the channel statistics of the player as well as that of others. Another related question: what if the BS does not know whether a mobile cooperates or not?

Finally, it should be clear that our approach is applicable not just to wireless networks, but is equally applicable to other resource allocation situations as for example in wireline networks.

\section{REFERENCES}

[1] Qualcomm, Inc., "1xEV:1x Evolution IS-856 TIA/EIA Standard Airlink Overview", Nov. 2001.
[2] 3GPP TS 25.308, Technical Specification 3rd Generation Partnership Project; Technical Specification Group Radio Access Network; High Speed Downlink Packet Access (HSDPA); iOverall description; Stage 2, Release 8, V8.3.0, sept 2008.

[3] R. Agrawal, A. Bedekar, R. J. La, and V. Subramanian, "Class and channel condition based weighted proportional fair scheduler,' in Teletraffic Engineering in the Internet Era, Proc. ITC-17, S. da Bahia, J. M. de Souza, N. L. S. da Fonseca, and E. A. de Souza e Silva, Eds. Amsterdam, The Netherlands: North-Holland, 2001, pp. 553565.

[4] D. M. Andrews, K. Kumaran, K. Ramanan, A. L. Stolyar, R. Vijayakumar, and P. A. Whiting, "Scheduling in a queueing system with asynchronously varying service rates," Prob. Eng. Inf. Sci., vol. 18, pp. 191217, 2004.

[5] P. Bender, P. Black, M. Grob, R. Padovani, N. Sindhushayana, and A. Viterbi, "CDMA/HDR: A bandwidth-efficient high-speed wireless data service for nomadic users," IEEE Commun. Mag., vol. 38, no. 7, pp. 7077, July 2000.

[6] A. Benveniste, M. Mietivier and P. Priouret, "Adaptive algorithms and stochastic approximation", Springer-Verlag, April 1990.

[7] S. C. Borst and P. A. Whiting, "Dynamic rate control algorithms for HDR throughput optimization," in Proc. IEEE INFOCOM, 2001, pp. 976985.

[8] E. F. Chaponniere, P. J. Black, J. M. Holtzman, and D. N. C. Tse, "Transmitter Directed code division multiple access system using path diversity to equitably maximize throughput," U.S. Patent 6,449,490, Sep. 10, 2002.

[9] J. Farrell, "Meaning and credibility in cheap-talk games," Games and Economic Behavior, vol. 5, pp. 514-531, 1993,

[10] D. Garg, Y. Narahari, "Foundations of Mechanism Design," Technical Report, Dept. of Computer Science and Automation, Indian Institute of Science, Bangalore, India, Nov. 2006. Weblink: http://lcm.csa.iisc.ernet.in/gametheory/ survey/md_nov06.pdf

[11] V. Kavitha, E. Altman, R. El-Azouzi and R.Sundaresan, ”Opportunistic scheduling in cellular systems in the presence of noncooperative mobiles", Technical repport available at http://lia.univavignon.fr/fileadmin/documents/Users/Intranet/fich_art/elazouzi/ Scheduling.pdf

[12] V. Kavitha, E. Altman, R. El-Azouzi and R.Sundaresan, ”Opportunistic scheduling in cellular systems in the presence of noncooperative mobiles", Submitted to IEEE Trans Information theory.

[13] F. P. Kelly, "Charging and rate control for elastic traffic," Euro. Trans. on Telecommun., vol. 8, pp. 33-37, 1997.

[14] Z. Kong, Y-K. Kwok, and J. Wang, "On game theoretic ratemaximizing packet scheduling in non-cooperative wireless networks," in Proc. IEEE Int. Symp. on a World of Wireless, Mobile and Multimedia Networks 2007, WoWMoM 2007, pp. 1-4, June 2007.

[15] D. M. Kreps and J. Sobel, "signaling", Chapter 25 in Handbook of Game Theory, vol. 2, R. J. Aumann and S. Hart, Elsevier Science B.V., 1994, pp. 849-867.

[16] H.J. Kushner, P.A. Whiting, "Convergence of Proportional-Fair Sharing algorithms under general conditions," IEEE Trans. Wireless Commun. vol. 3, no. 4, pp. 12501259, Jul. 2004.

[17] X. Liu, E. K. P. Chong, and N. B. Shroff, "A framework for opportunistic scheduling in wireless networks," Comput. Netw., vol. 41, pp. 451474, 2003.

[18] P. Nuggehalli, M. Sarkar, K. Kulkarni, R. R. Rao, "A game-theoretic analysis of QoS in wireless MAC," Proc. IEEE INFOCOM, pp. 1903-1911, Apr. 2008.

[19] A. Ozdaglar and R. Srikant, "Incentives and pricing in communications networks," Chapter 22 in Algorithmic Game Theory, N. Nisan, T. Roughgarden, E. Tardos, and V. V. Vazirani, pp. 571 - 591, 2007.

[20] J. Price and T. Javidi, "Leveraging downlink for efficient uplink allocation in a single-hop wireless network," IEEE Trans. Inform. Th., vol. 53, pp. 4330-4339, Nov. 2007.

[21] S. Shakkottai and A. L. Stolyar, "Scheduling algorithms for a mixture of real-time and non-real-time data in HDR," in Teletraffic Engineering in the Internet Era, Proc. ITC-17, S. da Bahia, J. M. de Souza, N. L. S. da Fonseca, and E. A. de Souza e Silva, Eds. Amsterdam, The Netherlands: North-Holland, 2001, pp. 793804.

[22] Joel Sobel, "Signaling Games, To appear in Encyclopedia of Complexity and System Science", M. Sotomayor (ed.), Springer, forthcoming, 2009. 